\title{
Design of Non-Circular Pulleys for Torque Generation: A Convex Optimisation Approach
}

\author{
Daniele Ludovico ${ }^{\circledR}$, Paolo Guardiani ${ }^{\circledR}$, Francesco Lasagni, Jinoh Lee ${ }^{\circledR}$, Ferdinando Cannella, \\ and Darwin G. Caldwell ${ }^{\circ}$
}

\begin{abstract}
Nowadays, robotic research focuses more and more on attaining energy-efficient and safe solutions. They are key-aspects of industrial robots, such as inspection and maintenance robots. The introduction of a mechanism that passively compensates the joint torque caused by the weight of the robot may offer a valid solution. Avoiding the need for actuators to balance gravity torques helps decrease the power consumption and the size of the actuators. Furthermore, a passive gravity compensation mechanism allows the robot to hold a static position without the need for an external power source, hence avoiding the risk of collapsing in case of failure of the actuators. This work focuses on designing a torque generator composed of a non-circular pulley and a spring, which, by solving a convex optimisation problem, offers a new methodology for creating any generic torque and thereby also succeeds in solving gravity compensation problems. This methodology guarantees the outcome of feasible non-circular pulleys which minimise the torque required to perform any specific task.
\end{abstract}

Index Terms-Mechanism design, methods and tools for robot system design.

\section{INTRODUCTION}

$\mathbf{U}$ P TO NOW, most of the classical industrial robots are designed to be perfectly rigid, to withstand high payloads and accelerations, move at high velocity, and ensure precision and repeatability of their movements. Achieving these performances comes at the cost of having a bulkier and, above all, heavier structure.

Another prerogative of classical industrial robots, particularly in robot manipulators, is good manipulability and dexterity, which can be achieved through a redundant kinematic chain. The increment of the torque due to the weight of a heavier, stiffer, and

Manuscript received September 22, 2020; accepted January 22, 2021. Date of publication February 2, 2021; date of current version February 15, 2021 This letter was recommended for publication by Associate Editor S. Revzen and Editor C. Gosselin upon evaluation of the reviewers' comments. (Corresponding author: Daniele Ludovico.)

Daniele Ludovico and Paolo Guardiani are with the Department of Informatics, Bioengineering, Robotics, and System Engineering, University of Genoa, Genoa, GE 16126, Italy, and also with the Advanced Robotics Department, Istituto Italiano di Tecnologia (IIT), Genoa, GE 16163, Italy (e-mail: daniele.ludovico@iit.it; paolo.guardiani@iit.it).

Ferdinando Cannella and Darwin G. Caldwell are with the Advanced Robotics Department, Istituto Italiano di Tecnologia (IIT), Genoa, GE 16163, Italy (e-mail: ferdinando.cannella@iit.it; Darwin.Caldwell@iit.it).

Francesco Lasagni is with the Politecnico di Torino, Torino 10129, Italy (email: francescolasagni93@gmail.com).

Jinoh Lee was with the Advanced Robotics Department, Istituto Italiano di Tecnologia (IIT), Genoa 16163, Italy. He is now with the Institute of Robotics and Mechatronics, German Aerospace Center (DLR), Weßling 82234, Germany (e-mail: jinoh.lee@dlr.de).

Digital Object Identifier 10.1109/LRA.2021.3056358 longer structure, causes the need for more powerful actuators in order to overcome the pull of gravity. In turn, a large actuator adds weight to the robot and implies a higher power consumption to keep static balance.

In this scenario, the study of mechanisms reducing static force is crucial to increasing energy efficiency, improving dynamic response, and carrying a heavier payload. Different types of weight compensation mechanisms are presented and categorised in [1]. The counterweight mechanism in [2] allows moving the centre of mass of each link to the joint axis of rotation. This technique allows smooth dynamic behaviours but has the drawback of increasing the total inertia and volume of the system.

Authors in [3] propose a magnetic balancing mechanism by exploiting the fact that two nested Halbach cylinders produce a sinusoidal torque enabling the compensation of that produced by a gravitational load over a complete rotation of a revolute joint. Even if this solution is difficult to finely tune and obtaining perfect compensation is not possible, it has the advantages of being extremely compact with the cylinders mounted in axis with the joint as well as the possibility to be modular; several modules can thus be combined in series to increasing torques.

Spring mechanisms are widely studied because they add less inertia than counterweight and are simpler to implement than the magnetic mechanism, but, on the other side, it is more difficult to perfectly match the non-linear joint torque induced by gravity and to obtain a smooth dynamic behaviour.

Torsional springs are rarely used compared to axial ones since it is more complex to adapt and adjust their behaviour to achieve static balance [4]. Radaelli et al. obtain good results for balancing an inverted pendulum with pre-stressed torsion bars [5].

The use of axial springs is well-documented in [6]. There are different ways to connect the spring to the system to compensate for the gravity torques. The simplest one is to connect the springs directly to the manipulator links. However, this technique leads to an exact compensation only if a zero-free length spring is used, and even in this case, it is complex to fine-tune the mechanism [7]. Besides, there is also the risk that the protruded spring may interfere with other parts of the robot. To improve the gravity compensation of the before-mentioned mechanisms Arakelian et al. introduce auxiliary links to minimise the residual unbalance [8]. Kim et al. in [9] design a device partially compensating the torque produced by gravity force on a 6-DOF manipulator using a linear spring. Axial springs have been recently applied to compensate for the gravity torque acting on robotic waists. Yun et al. designed a 3-DOF mechanism capable of compensating up to $23 \mathrm{~kg}$ load [10]. Reinecke et al. presented 
a 2-DOF device able to balance the weight of the upper body of a robot that moves dynamically [11].

More elaborated techniques involving cables, additional links, pulleys, and cams solve the problems related to zero-free length spring and interference with other parts of the mechanism. For example, a pulley and a cable can be arranged to simulate a zero-free length spring by storing away the initial length of the spring [12], [13]. Nguyen et al. in [14], [15] were able to considerably reduce the peak torque produced by gravity force acting on a planar articulated robotic arm and a parallel delta robot employing gear-spring modules.

Pulleys and cams with non-circular shapes increase the number of design parameters in the system, simplifying the optimisation of gravity compensation mechanisms [16]-[18]. Fedorov et al. proposed mechanisms based on a pair of differential non-circular pulleys to generate non-monotonic torque profiles through a geometrical method [19]. In [20], Kim et al. defined an analytical solution to find the shape of non-circular pulleys employed to generate an arbitrary torque profile. One of the limits of this work is that the authors did not consider all the constraints on the feasibility of the pulley. In particular, they did not count the potential presence of non-regular points [21], where the rope cannot perfectly wrap the pulley. Furthermore, during the design phase, evaluating constraints is not possible, so it is necessary to iterate the design by tuning some parameters, such as the spring stiffness, the spring initial length, or the insertion point, until a feasible solution is obtained.

This letter proposes a convex optimisation formulation of the pulley profile generation to overcome these limitations. First, in Section II, the analytical solution derived in [20] is revisited as a baseline. Section III presents the proposed convex optimisation method defining the cost function and detailing how to obtain the convex formulation of the constraints. Two design examples are shown in Section IV to compare the analytical solution with the convex optimisation solution.

\section{BASELINE: ANALYTIC SOLUTION FOR NON-CIRCULAR Pulleys DESIGN}

Consider the mechanism represented in Fig. 1, where $O$ is the origin of the reference frame $O_{x y}$. The pulley, body $B_{1}$, is fixed to the ground. On one side, the linear extension spring is attached to the link, body $B_{2}$, at insertion point $R$. On the other side, it is fixed at the anchor point $A$ through a cable that wraps around the pulley. $r_{m}(\theta)$ represents the moment arm that, multiplied by the spring force at joint position $\theta$, determines the torque applied to the joint. The spring force line of action is tangent to the pulley and passes through the insertion point $R$ and the point $Q$. The point $P$ represents the tangent point between $\overline{R Q}$ and the pulley. The angle formed by the intersection of the direction of the spring force and the line passing through the point $O$ and $R$ is called $\phi$.

Given the spring stiffness, and its initial length, the pulley profile is determined in closed form, as shown in [20] under the assumptions that the spring behaviour is linear, no pulley is present on the insertion point, and the cable is rigid with negligible diameter. The derivation of the closed form solution,

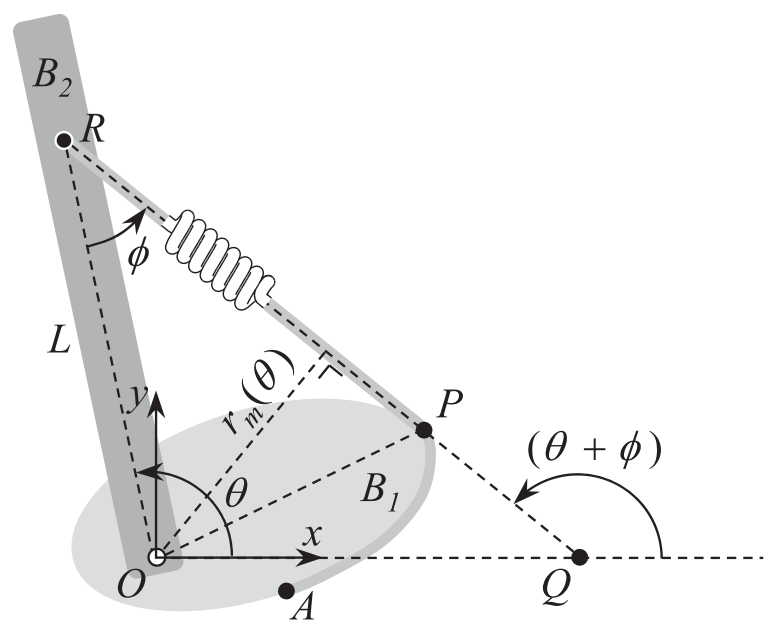

Fig. 1. Schematic diagram of a mechanical system with a spring and a non circular pulley.

discussed in detail in [20], is summarised as follows:

$$
\begin{aligned}
& r_{m}(\theta)=\frac{\tau_{d}(\theta)}{\sqrt{2 k \int_{\theta_{0}}^{\theta} \tau_{d}(\alpha) d \alpha+k^{2} u_{0}^{2}}}, \\
& \phi=\sin ^{-1}\left(\frac{r_{m}(\theta)}{L}\right), \\
& S(\theta)=\tan (\theta+\phi), \\
& Y(\theta)=L[\sin \theta-\cos \theta \cdot \tan (\theta+\phi)], \\
& \left(x_{p}(\theta), y_{p}(\theta)\right)=\left(-\frac{Y^{\prime}(\theta)}{S^{\prime}(\theta)}, Y(\theta)-\frac{Y^{\prime}(\theta)}{S^{\prime}(\theta)} S(\theta)\right),
\end{aligned}
$$

where $x_{p}$ and $y_{p}$ are $x$ - and $y$-Cartesian coordinates of the pulley profile represented in the frame $O_{x y}$, respectively.

However, it is worth noticing that the pulley shape obtained through this method could be unrealistic. As discussed in previous works [20], [22], to get a feasible pulley, it is necessary to avoid that the radius of the pulley contour goes to infinite, and the shape must be convex. This condition can be translated into a constraint on the curvature of the profile as follows:

$$
1+\phi^{\prime}(\theta) \neq 0 \text {. }
$$

Besides, the pulley profile cannot have non-regular points because, in these points, the rope cannot wrap perfectly. This condition is satisfied if the zeros of $x_{P}^{\prime}(\theta)$ are different from the zeros of $y_{P}^{\prime}(\theta)$.

\section{PROPOSED METHOD BASED ON CONVEX OPTIMISATION}

In this section, a convex optimisation problem to compute the moment arm $r_{m}(\theta)$ as its solution, guaranteeing the feasibility of the pulley shape, has been formulated. This approach enables conditions on the pulley profile to be included as constraints of the optimisation problem and promises a feasible shape at the expense of introducing an error in the torque generation.

\section{A. Cost Function Definition}

The objective function of the optimisation problem is derived directly from (1). The angle $\theta$ is discretised over the range of 
motion of the joint from the initial angle $\theta_{i}$ to the final $\theta_{f}$, given by

$$
\boldsymbol{\theta} \in \mathbb{R}^{\mathrm{n}}: \boldsymbol{\theta}=\left[\begin{array}{llll}
\theta_{0} & \theta_{1} \ldots \theta_{n-1}
\end{array}\right]^{\mathrm{T}} .
$$

Without loss of generality it is possible to parametrise the moment arm as a polynomial of order $m$ as follows:

$$
\begin{aligned}
\boldsymbol{r}_{m} & =\beta_{0} \mathbb{1}+\beta_{1} \boldsymbol{\theta}+\beta_{2} \boldsymbol{\theta}^{2}+\cdots+\beta_{m} \boldsymbol{\theta}^{m} \\
& =\left[\begin{array}{lllll}
\mathbb{1} & \boldsymbol{\theta} & \boldsymbol{\theta}^{2} & \ldots & \boldsymbol{\theta}^{m}
\end{array}\right] \cdot \boldsymbol{\beta} \\
& =H \cdot \boldsymbol{\beta} .
\end{aligned}
$$

Define the angle increment as $d \alpha=\frac{\theta_{f}-\theta_{i}}{n}$ and the vector $\boldsymbol{v}$ as

$$
\boldsymbol{v} \in \mathbb{R}^{\mathrm{n}}: \mathrm{v}_{\mathrm{i}}=\sqrt{2 \mathrm{k} \sum_{\alpha=0}^{\mathrm{i}} \tau_{\mathrm{d}}\left(\theta_{\alpha}\right) \mathrm{d} \alpha+\mathrm{k}^{2} \mathrm{u}_{0}^{2}}, \quad \forall \theta_{\alpha} \in \boldsymbol{\theta} .
$$

Substituting $\boldsymbol{v}$ in (1) yields the moment arm equation rewritten as

$$
\operatorname{diag}(\boldsymbol{v}) \cdot(\mathrm{H} \cdot \boldsymbol{\beta})-\boldsymbol{\tau}_{\mathrm{d}}=0 .
$$

By squaring the $\ell_{2}$-norm of (9), one can then obtain a convex objective function which minimises the sum of the torque residuals squared as follows:

$$
f_{0}(\boldsymbol{\beta})=\left\|\operatorname{diag}(\boldsymbol{v}) \cdot(\mathrm{H} \cdot \boldsymbol{\beta})-\boldsymbol{\tau}_{\mathrm{d}}\right\|_{2}^{2} .
$$

\section{B. Constraints Definition}

1) Moment Arm Bounds: The moment arm $r_{m}(\theta)$ must be greater than 0 and smaller than the insertion length $L$. Otherwise, the mobile link would hit the non-circular pulley and there would be no space to insert the spring. These conditions can be written as follows:

$$
\begin{gathered}
\|H \cdot \boldsymbol{\beta}\|_{\infty}<L, \\
\|H \cdot \boldsymbol{\beta}\|_{\infty}>0 .
\end{gathered}
$$

2) Curvature Constraint: As discussed in Section II, to avoid collision between the cable and other parts of the non-circular pulley, the pulley shape needs to be convex. The straightforward way to satisfy this condition is to introduce a constraint on the curvature of the pulley, so that it does not change the sign.

Starting from (6) it is possible to write this constraint as

$$
\begin{aligned}
& 1+\phi^{\prime}>0 \cup 1+\phi^{\prime}<0 \Rightarrow \\
& 1+\frac{r_{m}^{\prime}}{\sqrt{L^{2}-r_{m}^{2}}}>0 \cup 1+\frac{r_{m}^{\prime}}{\sqrt{L^{2}-r_{m}^{2}}}<0 .
\end{aligned}
$$

The disequalities (13) can be written as

$$
\begin{aligned}
& r_{m}^{\prime}>-\sqrt{L^{2}-r_{m}^{2}} \cup r_{m}^{\prime}<-\sqrt{L^{2}-r_{m}^{2}} \\
& \Rightarrow\left\{\begin{array} { l } 
{ r _ { m } ^ { 2 } < L ^ { 2 } } \\
{ r _ { m } ^ { \prime } \leq 0 } \\
{ r _ { m } ^ { 2 } + r _ { m } ^ { \prime 2 } < L ^ { 2 } }
\end{array} \cup \left\{\begin{array} { l } 
{ r _ { m } ^ { 2 } < L ^ { 2 } } \\
{ r _ { m } ^ { \prime } \geq 0 }
\end{array} \cup \left\{\begin{array}{l}
r_{m}^{2}<L^{2} \\
r_{m}^{\prime} \leq 0 \\
r_{m}^{2}+r_{m}^{\prime 2}>L^{2} .
\end{array}\right.\right.\right.
\end{aligned}
$$

The set generated by (14) is not convex, as shown in Fig. 2 a. Many different convex subsets of (14) can be considered to obtain a convex formulation of the curvature constraint. The main effect of reducing the feasible set is not to reach the global optimum, but the optimal solution belonging to the convex subset

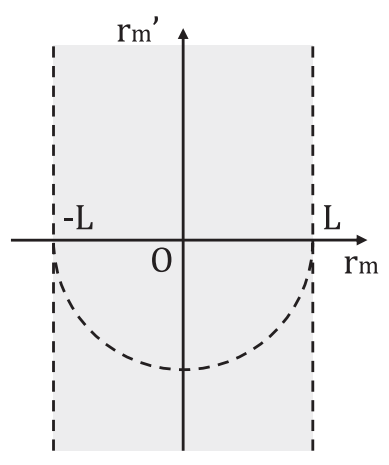

(a)

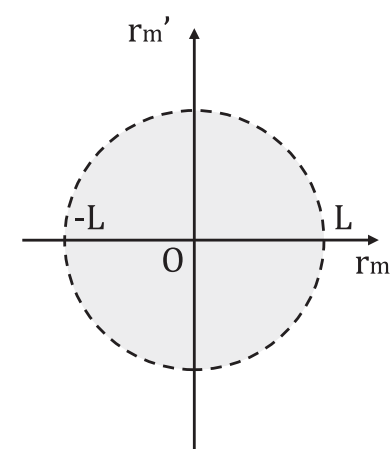

(b)
Fig. 2. Geometric representation of the curvature constraint: (a) the set defined by the curvature constraint (14); (b) the set defined by the convex approximation of the curvature constraint (14) described by (15). The dashed lines represent the points excluded in the set.

considered. A possible choice for the convex approximation of (14) is represented by

$$
r_{m}^{2}+r_{m}^{\prime 2}<L^{2}
$$

As shown in Fig. 2 b, the set described by (15) is convex and satisfies the constraint on the curvature of the pulley profile. Furthermore, choosing (15) as curvature constraint, the variation of the moment arm is bounded. This additional condition makes $r_{m}$ smooth allowing to obtain a pulley on which the rope can perfectly wrap.

As for $r_{m}, r_{m}^{\prime}$ can be written in terms of the discretised angle $\boldsymbol{\theta}$ and the design variables $\boldsymbol{\beta}$

$$
\begin{aligned}
& \boldsymbol{r}_{m}^{\prime}=\beta_{1} \mathbb{1}+2 \beta_{2} \boldsymbol{\theta}+3 \beta_{3} \boldsymbol{\theta}^{2}+\cdots+m \beta_{m} \boldsymbol{\theta}^{m-1} \\
& =\left[\begin{array}{llllll}
\mathbb{1} & \mathbb{1} & 2 \boldsymbol{\theta} & 3 \boldsymbol{\theta}^{2} & \ldots & m \boldsymbol{\theta}^{m-1}
\end{array}\right] \cdot \boldsymbol{\beta} \\
& =H_{d} \cdot \boldsymbol{\beta} \text {. }
\end{aligned}
$$

Finally, by substituting (16) into (15), the curvature constraint can be written as a set of quadratic constraints in $\boldsymbol{\beta}$ as follows:

$$
\boldsymbol{\beta}^{T} \cdot\left(H_{i}^{T} H_{i}+H_{d_{i}}^{T} H_{d_{i}}\right) \cdot \boldsymbol{\beta}<L, \quad i=1 \ldots n,
$$

where $H_{i}$ and $H_{d_{i}}$ are respectively the rows of $H$ and $H_{d}$. This type of constraint can be easily implemented using well-known convex optimisation algorithms.

3) Non-Regular Points Avoidance: Consider the parametric curve defined in (5)

$$
\gamma(\theta)=\left\{\begin{array}{l}
x_{p}(\theta)=-\frac{Y^{\prime}(\theta)}{S^{\prime}(\theta)} \\
y_{p}(\theta)=Y(\theta)-\frac{Y^{\prime}(\theta)}{S^{\prime}(\theta)} S(\theta) .
\end{array}\right.
$$

Non-regular points of $\gamma(\theta)$ are all the values of $\theta$ such that $\gamma^{\prime}(\theta)=\mathbf{0}$ [21].

The derivative of $\gamma(\theta)$ can be written as

$$
\gamma^{\prime}(\theta)=\left\{\begin{array}{l}
x_{p}^{\prime}(\theta)=-h(\theta) \\
y_{p}^{\prime}(\theta)=-h(\theta) S(\theta) .
\end{array}\right.
$$

where

$$
h(\theta)=\frac{Y^{\prime \prime} S^{\prime}-Y^{\prime} S^{\prime \prime}}{S^{2}} .
$$


From (3) and (4), one can compute $Y$ and its derivatives as

$$
\begin{aligned}
& Y=L[\sin (\theta)-\cos (\theta) S], \\
& Y^{\prime}=L\left[\cos (\theta)\left(1-S^{\prime}\right)+\sin (\theta) S\right], \\
& Y^{\prime \prime}=L\left[\sin (\theta)\left(2 S^{\prime}-1\right)+\cos (\theta)\left(S-S^{\prime \prime}\right)\right] .
\end{aligned}
$$

Substituting (20)-(22) into (19) gives $h$ as a function of $\theta, S, S^{\prime}$ and $S^{\prime \prime}$ only,

$$
h=L \frac{\sin (\theta)\left[2 S^{\prime 2}-S^{\prime}-S S^{\prime \prime}\right]+\cos (\theta)\left[S S^{\prime}-S^{\prime \prime}\right]}{S^{\prime 2}} .
$$

The points in which $\gamma^{\prime}(\theta)=\mathbf{0}$ are the zeros of the function $h(\theta)$. Since $S^{\prime 2}$ is always different from zero, and imposing $h(\theta)=0$, the following equation is obtained:

$$
\tan (\theta)=\frac{S S^{\prime}-S^{\prime \prime}}{2 S^{\prime 2}-S^{\prime}-S S^{\prime \prime}} .
$$

Moreover considering that

$$
\begin{aligned}
& S^{\prime}(\theta)=\left(1+S^{2}\right)\left(1+\phi^{\prime}\right), \\
& S^{\prime \prime}(\theta)=\left(1+S^{2}\right)\left[2 S\left(1+\phi^{\prime}\right)^{2}+\phi^{\prime \prime}\right],
\end{aligned}
$$

equation (24) can be rewritten as

$$
\tan (\theta)=\frac{\phi^{\prime \prime}+S g\left(\phi^{\prime}\right)}{-S \phi^{\prime \prime}+g\left(\phi^{\prime}\right)}
$$

where

$$
g\left(\phi^{\prime}\right)=\left(1+\phi^{\prime}\right)\left[2\left(1+\phi^{\prime}\right)-1\right] .
$$

By using (3) and (27), the following equation is obtained:

$$
\begin{aligned}
\phi^{\prime \prime} & =-g\left(\phi^{\prime}\right) \frac{\tan (\theta+\phi)-\tan (\theta)}{1+\tan (\theta+\phi) \tan (\theta+\phi)}= \\
& =-g\left(\phi^{\prime}\right) \tan (\phi) .
\end{aligned}
$$

Finally computing the derivatives of $\phi$ and substituting (2) and (28) into (29) gives

$$
r_{m}^{\prime \prime}+r_{m}\left(1+3 \frac{r_{m}^{\prime}}{\sqrt{L^{2}-r_{m}^{2}}}+3 \frac{r_{m}^{\prime 2}}{L^{2}-r_{m}^{2}}\right)=0
$$

Considering that

$$
1+3 \frac{r_{m}^{\prime}}{\sqrt{L^{2}-r_{m}^{2}}}+3 \frac{r_{m}^{\prime 2}}{L^{2}-r_{m}^{2}} \geq \frac{1}{4}
$$

it is possible to write the following inequality:

$$
r_{m}^{\prime \prime}+r_{m}\left(1+\frac{3 r_{m}^{\prime}}{\sqrt{L^{2}-r_{m}^{2}}}+\frac{3 r_{m}^{\prime 2}}{L^{2}-r_{m}^{2}}\right) \geq r_{m}^{\prime \prime}+\frac{1}{4} r_{m} .
$$

Equation (32) demonstrates that

$$
r_{m}^{\prime \prime}+\frac{1}{4} r_{m}>0 \Rightarrow h(\theta) \neq 0 .
$$

Note that $r_{m}^{\prime \prime}$ and $r_{m}$ can be written in terms of the discretised angle $\boldsymbol{\theta}$ and the design variables $\boldsymbol{\beta}$ as

$$
\begin{aligned}
\boldsymbol{r}_{m}^{\prime \prime} & =2 \beta_{2} \mathbb{1}+6 \beta_{3} \boldsymbol{\theta}+\cdots+m(m-1) \beta_{m} \boldsymbol{\theta}^{m-2} \\
& =\left[\begin{array}{llllll}
\mathbb{1} & \mathbb{1} & 2 \cdot \mathbb{1} & 6 \boldsymbol{\theta} & \ldots & m(m-1) \boldsymbol{\theta}^{m-2}
\end{array}\right] \cdot \boldsymbol{\beta} \\
& =H_{d d} \cdot \boldsymbol{\beta} .
\end{aligned}
$$

Accordingly, to avoid non-regular points in the pulley shape, it is enough to add the following linear constraints:

$$
\left\|\left(H_{d d}+\frac{1}{4} H\right) \cdot \boldsymbol{\beta}\right\|_{\infty}>0 .
$$

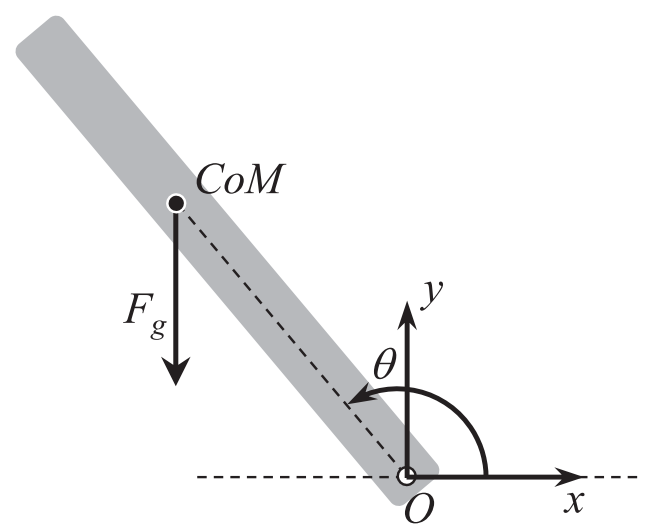

Fig. 3. Schematic of the force acting on an inverted pendulum.

\section{Optimisation Problem}

With considering the cost function (10) and constraints (11), (12), (17) and (35), the problem of designing a non-circular pulley for torque generation can be solved as the convex optimisation problem described by

$$
\begin{aligned}
\min _{\boldsymbol{\beta}} & \left\|\operatorname{diag}(\boldsymbol{v}) \cdot(\mathrm{H} \cdot \boldsymbol{\beta})-\boldsymbol{\tau}_{\mathrm{d}}\right\|_{2}^{2} \\
\text { s.t. } & \|H \cdot \boldsymbol{\beta}\|_{\infty}>0 \\
& \|H \cdot \boldsymbol{\beta}\|_{\infty}<L \\
& \left\|\left(H_{d d}+\frac{1}{4} H\right) \cdot \boldsymbol{\beta}\right\|_{\infty}>0 \\
& \boldsymbol{\beta}^{T} \cdot\left(H_{i}^{T} H_{i}+H_{d_{i}}^{T} H_{d_{i}}\right) \cdot \boldsymbol{\beta}<L \quad i=1 \ldots N .
\end{aligned}
$$

In this optimisation problem, the cost function is quadratic, and the constraints are linear or quadratic inequalities. This family of convex problems can be solved as a semidefinite program using robust and efficient algorithms.

\section{DESIGN EXAMPLES}

In this section, the comparison between the analytical and the proposed convex optimisation solution is presented through two examples.The first describes the design of non-circular pulleys for compensating the gravity force acting on an inverted pendulum. The second presents the design of non-circular pulleys able to produce a generic torque profile. This example highlights the benefit introduced by the convex optimisation approach. The design algorithm is implemented in MATLAB, and the optimisation problem is solved using the CVX toolbox [23], [24].

\section{A. Gravity Compensation of the Inverted Pendulum}

In this example, the inverted pendulum presented in Fig. 3 is taken into account. The desired torque for compensating the gravity force acting on this mechanism is represented in Fig. 4 and is defined as follows:

$$
\tau_{d}(\theta)=\tau_{\max } \cos (\theta),
$$

where $\tau_{\max }=F_{g} \overline{O G}$ 


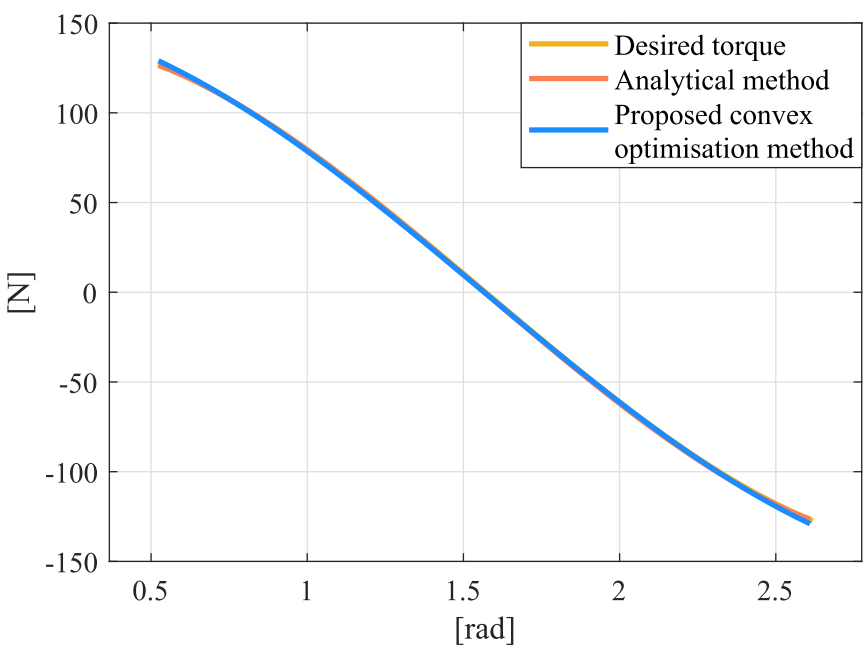

Fig. 4. Comparison of the desired torque with the torque produced by the analytical solution and the torque produced by the proposed convex optimisation solution.

TABLE I

INVERTED PENDULUM EXAMPLE PARAMETERS

\begin{tabular}{llcl}
\hline \hline Symbol & Value & Unit & Description \\
\hline$M$ & 15 & $\mathrm{Kg}$ & Pendulum mass \\
$l$ & 1 & $\mathrm{~m}$ & Pendulum length \\
$\tau_{\max }$ & 147.1 & $\mathrm{Nm}$ & Maximum desired torque \\
$d_{\text {off }}$ & 79.6 & $\mathrm{Nm}$ & Torque offset \\
$\lambda$ & 0.5 & - & Splitting torque coefficient \\
$k$ & $\left(30 \cdot 10^{3}\right)^{*}$ & $\mathrm{~N} / \mathrm{m}$ & Spring stiffness \\
$u_{0}$ & 0.015 & $\mathrm{~m}$ & Spring initial elongation \\
$L$ & 0.25 & $\mathrm{~m}$ & Spring insertion point \\
$\theta_{i}$ & $\pi / 6$ & $\mathrm{rad}$ & Initial angle \\
$\theta_{f}$ & $5 / 6 \pi$ & $\mathrm{rad}$ & Final angle \\
$m$ & 5 & - & Cost function polynomial order \\
$d \alpha$ & $8.378 \cdot 10^{-3}$ & $\mathrm{rad}$ & Motion range discretisation \\
\hline \hline
\end{tabular}

${ }^{*}$ Considerations about spring selection are detailed in the appendix.

As described in [20], to generate a bidirectional torque, two antagonistic pulleys are necessary. The desired torque has to be divided into two different sub-profiles to compensate for the gravity force acting on the pendulum in the range of motion $\left[\theta_{i}, \theta_{f}\right]$. Table I lists the parameters selected to define the torque sub-profiles.

Figure 5 presents both profiles obtained by the analytical and convex optimisation solution. The algorithm provides only the profile points generated by the angles belonging to the desired range of motion. Accordingly, the two boundary points have to be connected by any smooth curve to produce the entire profile. The light blue shape in Fig. 5 shows a possible complete pulley profile for the convex optimisation solution.

The symmetry of $\tau_{d}$ and the choice of the splitting torque coefficient $\lambda=0.5$ entail that the profile of the two antagonistic pulleys is symmetric, as explained in [20].

The design result highlights that the proposed algorithm generates a pulley profile able to approximate the desired torque with a maximum error of $2.2 \mathrm{Nm}$ while the analytical solution has a maximum error of $0.9 \mathrm{Nm}$ approximating better the desired

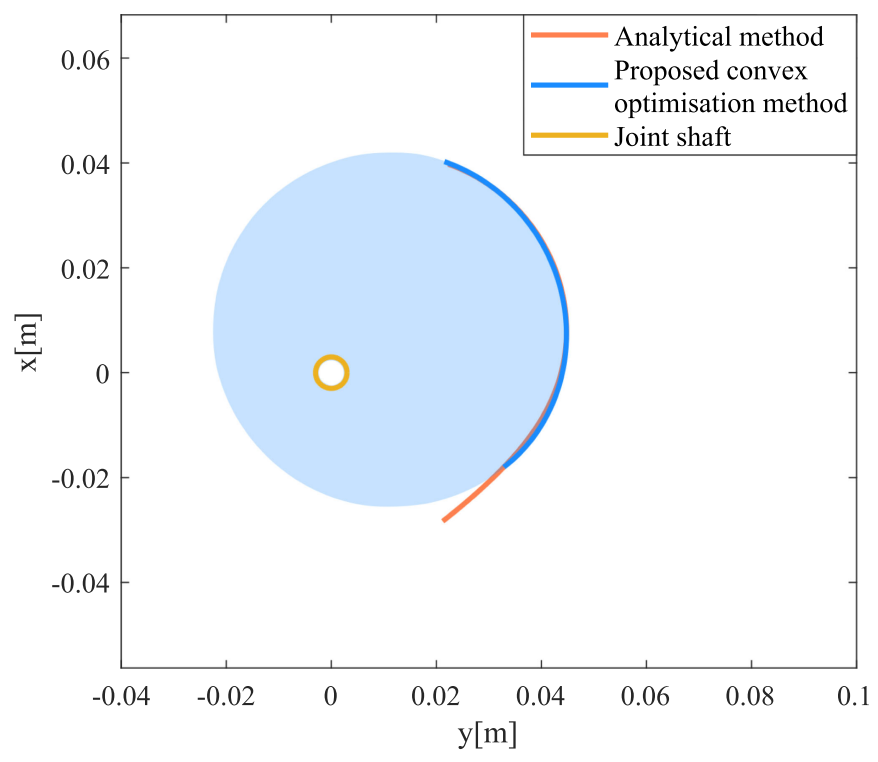

Fig. 5. Pulley shape for the inverted pendulum. The blue line represents the shape obtained with the analytical solution. The red line represents the convex optimisation solution. The complete pulley shape is represented in light blue for the convex optimisation solution.

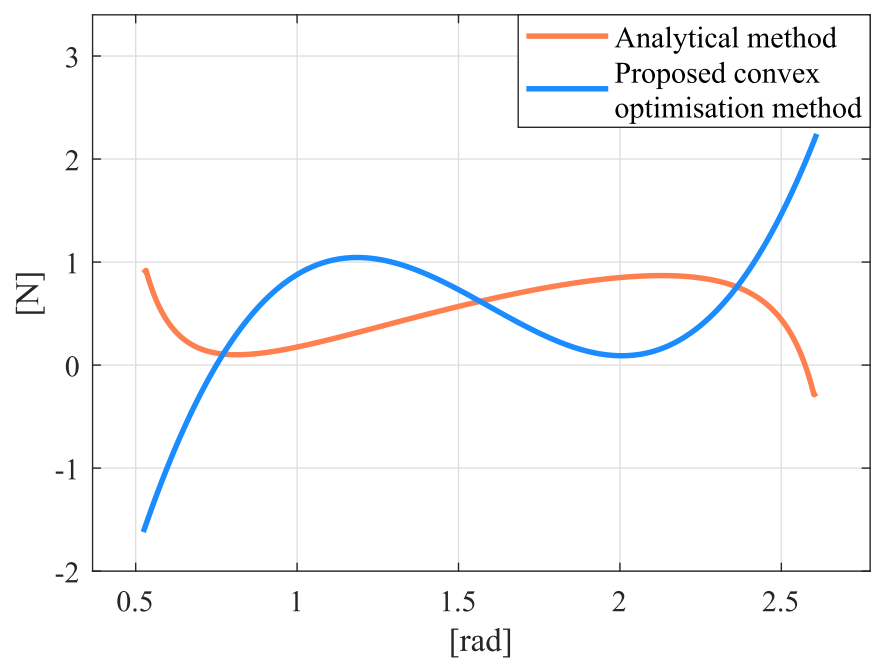

Fig. 6. Torque error produced by the analytical and the convex optimisation solution for the inverted pendulum.

torque. Figure 6 depicts the torque error produced by both the solutions.

To better evaluate the results, the error has been put in perspective with the desired torque. The percentage error is computed as the relative percent difference as follows:

$$
e_{\%}=\left(2 \frac{\tau_{d}-\tau}{\left|\tau_{d}\right|+|\tau|}\right) \times 100
$$

This formulation allows to bound the value of the percentage error in the range [ $[-200200]$ even if the desired torque is equal to zero. Table II compares the absolute and percentage error, respectively $e_{a b s}$ and $e_{\%}$, for both the analytical and the proposed convex optimisation method in different joint configurations. When $\theta=\pi / 2$, the desired torque is zero, so the percentage 
TABLE II

TORQUE ERROR FOR THE INVERTED PENDULUM EXAMPLE

\begin{tabular}{c|c|c|c|c}
\hline \hline \multirow{2}{*}{ Angle [rad] } & \multicolumn{2}{|c|}{ Analytical solution } & \multicolumn{2}{c}{ Convex solution } \\
\cline { 2 - 5 } & $e_{a b s}[\mathrm{Nm}]$ & $e_{\%}$ & $e_{a b s}[\mathrm{Nm}]$ & $e_{\%}$ \\
\hline \hline$\pi / 6$ & 0.9 & 0.7 & -1.6 & -1.2 \\
$\pi / 4$ & 0.1 & 0.1 & 0.2 & 0.2 \\
$\pi / 3$ & 0.2 & 1.1 & 0.9 & 1.0 \\
$5 \pi / 12$ & 0.4 & 2.0 & 1.0 & 2.6 \\
$\pi / 2$ & 0.6 & $(200.0)^{*}$ & 0.6 & $(200.0)^{*}$ \\
$7 \pi / 12$ & 0.8 & 1.2 & 0.2 & 0.5 \\
$2 \pi / 3$ & 0.9 & 0.7 & 0.1 & 0.2 \\
$3 \pi / 4$ & 0.8 & -0.2 & 0.7 & 0.7 \\
$5 \pi / 6$ & 0.3 & -1.2 & 2.2 & 1.8 \\
\hline \hline
\end{tabular}

${ }^{*}$ Bounded to the maximum value since the desired torque is zero.

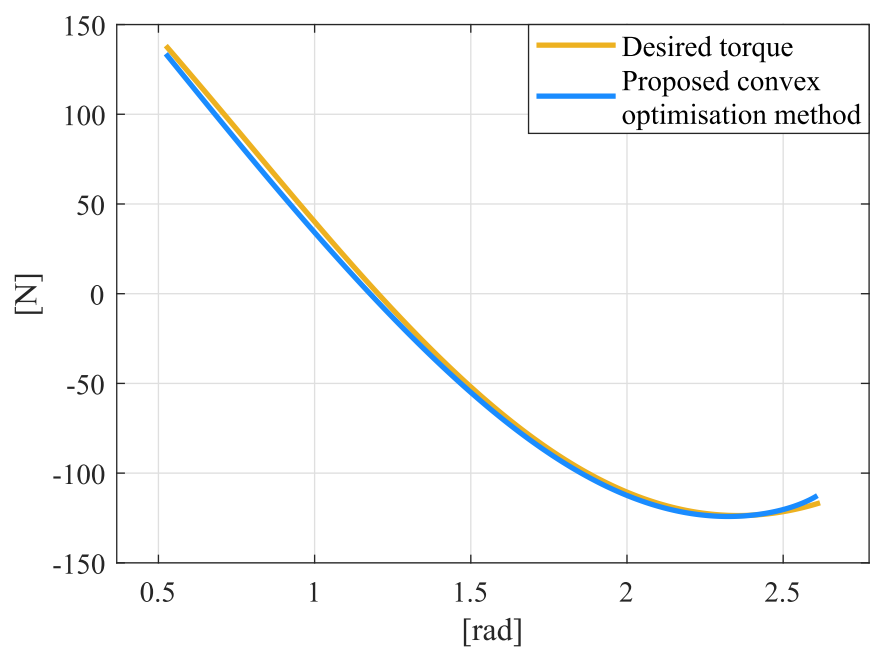

Fig. 7. Profile of the desired torque (39) and the torque generated by the convex optimisation solution.

error reaches the maximum value. Nevertheless, the residual torque is about $0.6 \mathrm{Nm}$.

\section{B. Generic Torque Profile Creation}

To verify that the proposed convex optimisation method always finds a feasible solution, in this example, the generic torque, represented in Fig. 7 and defined as

$$
\tau_{d}(\theta)=a(\cos (\theta)-\sin (\theta))+c,
$$

is considered.

Since $\tau_{d}$ is not symmetric, both the analytical and convex optimisation methods generate two antagonistic pulleys with different profiles. Table III lists the selected parameters to designing the pulleys. In this torque profile, the analytical solution produces an unfeasible shape for both the antagonistic pulleys. The first pulley does not satisfy the curvature constraints, whereas the second contains a non-regular point. Since the two pulleys have unfeasible shapes, the length of the cables wrapping on the pulleys is not defined, making it impossible to compute the torque produced by the analytical solution.

The convex optimisation approach, instead, can satisfy all the constraints producing feasible shapes. Figure 8 shows one of the
TABLE III

GENERIC TORQUE EXAMPLE PARAMETERS

\begin{tabular}{llcl}
\hline \hline Symbol & Value & Unit & Description \\
\hline$a$ & 147.1 & $\mathrm{Nm}$ & Torque parameter \\
$c$ & 84.4 & $\mathrm{Nm}$ & Torque parameter \\
$d_{\text {off }}$ & 86.4 & $\mathrm{Nm}$ & Torque offset \\
$\lambda$ & 0.5 & - & Splitting torque coefficient \\
$k$ & $30 \cdot 10^{3}$ & $\mathrm{~N} / \mathrm{m}$ & Spring stiffness \\
$u_{0}$ & 0.015 & $\mathrm{~m}$ & Spring initial elongation \\
$L$ & 0.25 & $\mathrm{~m}$ & Spring insertion point \\
$\theta_{i}$ & $\pi / 6$ & $\mathrm{rad}$ & Initial angle \\
$\theta_{f}$ & $5 / 6 \pi$ & $\mathrm{rad}$ & Final angle \\
$m$ & 5 & - & Cost function polynomial order \\
$d \alpha$ & $8.378 \cdot 10^{-3}$ & $\mathrm{rad}$ & Motion range discretisation \\
\hline \hline
\end{tabular}

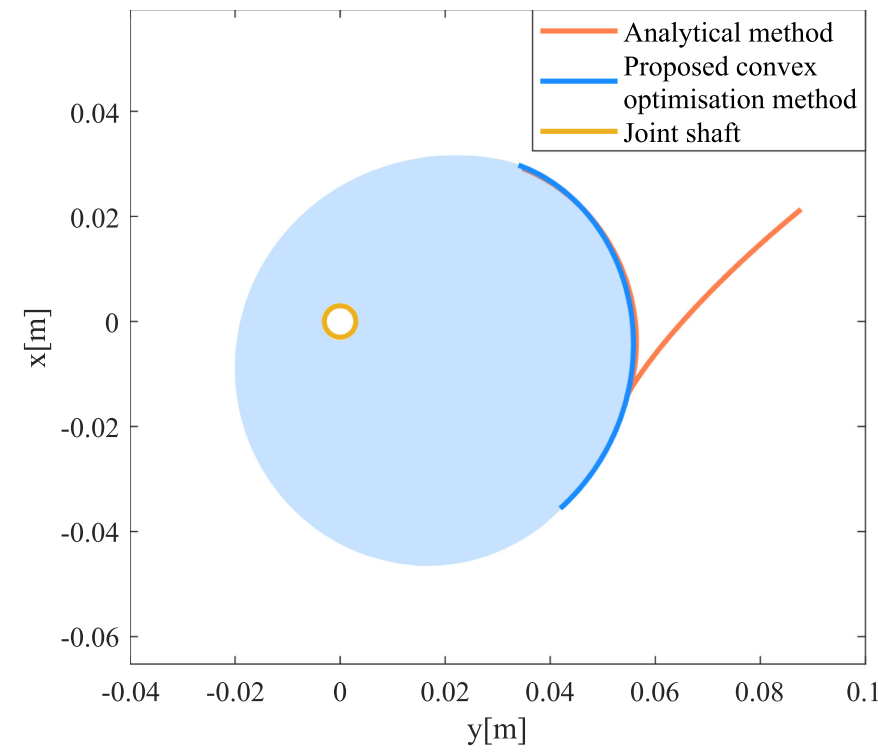

Fig. 8. Pulley shape to produce the generic torque (39). The blue line represents the shape obtained with the analytical solution. The red line represents the convex optimisation solution. The complete pulley shape is represented in light blue for the convex optimisation solution.

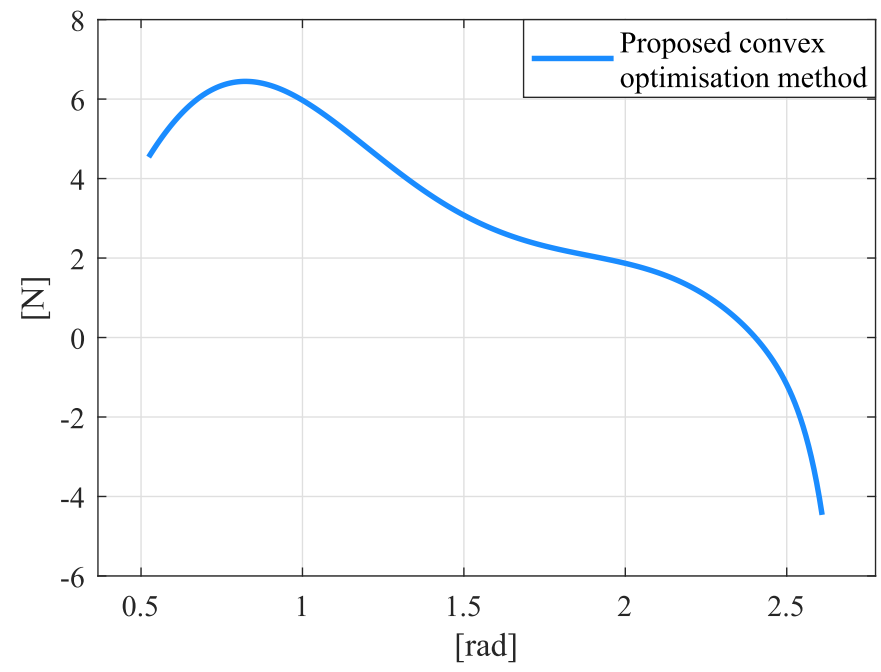

Fig. 9. Torque error produced by the convex optimisation solution for the generic torque (39). 
TABLE IV

TORQUE ERROR FOR THE GENERIC TORQUE EXAMPLE

\begin{tabular}{c|c|c}
\hline \hline \multirow{2}{*}{ Angle [rad] } & \multicolumn{2}{|c}{ Convex solution } \\
\cline { 2 - 3 } & $e_{\text {abs }}[\mathrm{Nm}]$ & $e_{\%}$ \\
\hline \hline$\pi / 6$ & 4.5 & 3.3 \\
$\pi / 4$ & 6.4 & 7.9 \\
$\pi / 3$ & 5.7 & 20.4 \\
$5 \pi / 12$ & 4.1 & 19.5 \\
$\pi / 2$ & 2.8 & 4.4 \\
$7 \pi / 12$ & 2.1 & 2.2 \\
$2 \pi / 3$ & 1.7 & 1.4 \\
$3 \pi / 4$ & 0.4 & 0.3 \\
$5 \pi / 6$ & -4.5 & -3.9 \\
\hline \hline
\end{tabular}

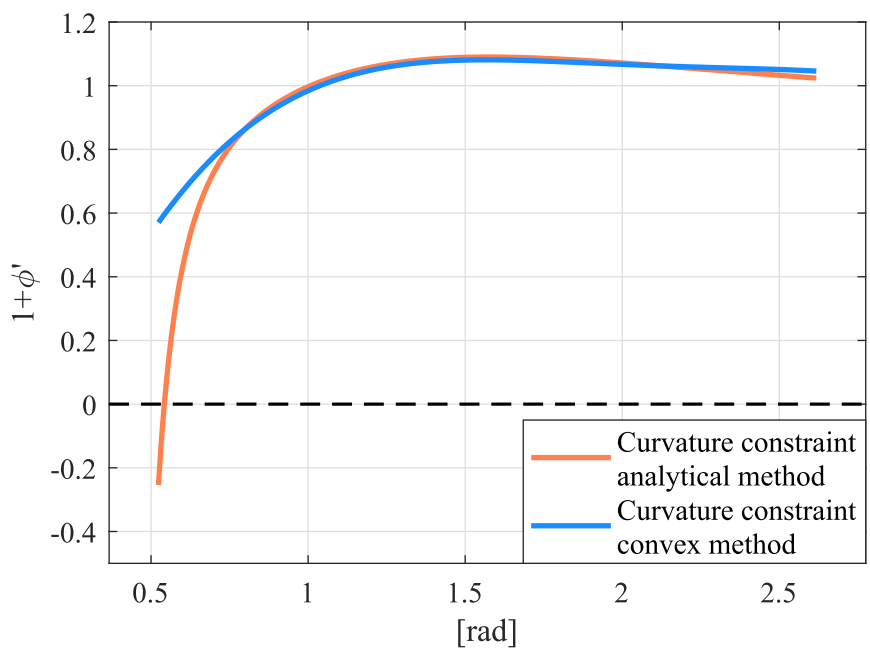

Fig. 10. Graphical representation of curvature constraint for both the analytical and convex optimisation solution.
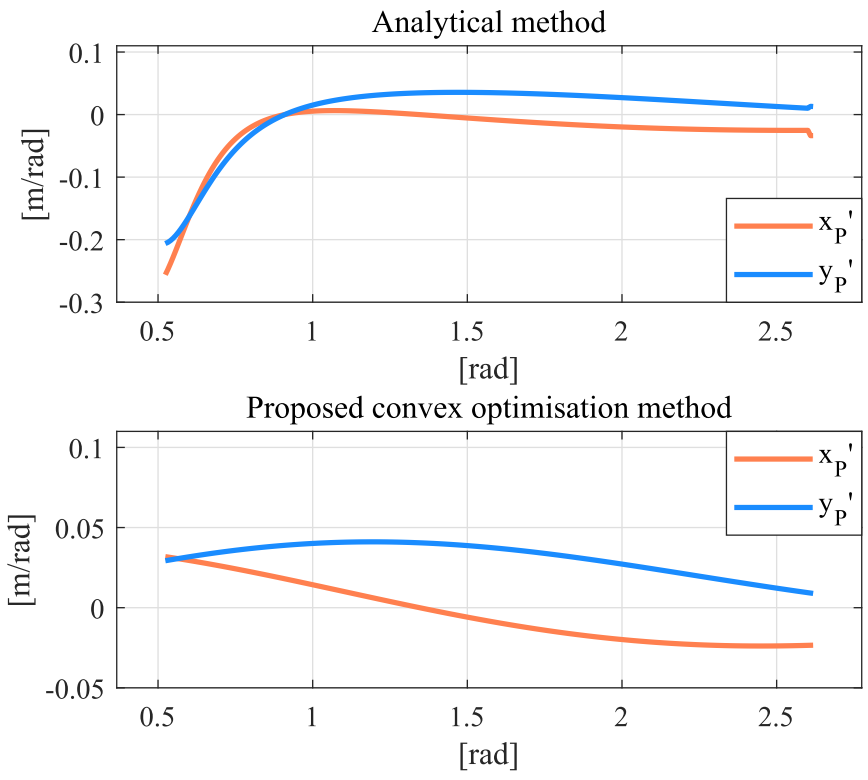

Fig. 11. Graphical representation of non-regular point constraint for both the analytical and convex optimisation solution. two pulley profile obtained by both the convex optimisation and the analytical solution where the presence of a non-regular point is evident.

The two antagonist pulleys, generated by the convex optimisation solution, can approximate the desired torque with a maximum error of $6.4 \mathrm{Nm}$, as shown in Fig. 9. Percentage errors are given in Table IV.

The curvature constraint for both analytical and convex optimisation solution is shown in Fig. 10, where it is possible to notice that the analytical solution has a zero in $\theta=0.54 \mathrm{rad}$, violating the constraint. In Fig. 11, the non-regular point constraint is represented for both the analytical and convex optimisation solution. In the analytical solution appears a non-regular point in $\theta=0.91 \mathrm{rad}$.

\section{CONCLUSION AND FUTURE WORKS}

This letter presented a convex optimisation approach to designing non-circular pulleys for torque generation. First, the analytical solution was presented. Then, the problem was framed as a convex optimisation problem and two different designs were given as examples. In the first example, a gravity compensation mechanism for an inverted pendulum was presented. Although the analytical solution provided a better approximation of the desired torque, the convex solution returned a good estimate of the desired torque, too. For the second example, a generic torque was required. In this case, the analytical solution was not able to generate a feasible shape, while the convex approach provided a good approximation of the desired torque.

In general the analytical method needs a long tuning procedure to set the parameters, without any guarantee of finding a feasible solution. The proposed convex optimisation solution overcomes this limitation by considering all the constraints right from the start, including the non-regular points constraint, while always returning a feasible pulley shape and obtaining an acceptable error with respect to the desired torque.

In future work, we plan to build a prototype of the non-circular pulley torque generator to validate the proposed method and to analyse the advantages in terms of mass reduction, energy consumption, and safety of the mechanism on a real device.

Furthermore, convex optimisation problems can easily be solved in real-time. This feature can be exploited to develop an active pulley able to adapt its shape, allowing, for example, to compensate the gravity acting on an articulated mechanism such as a robotic manipulator in which the torque depends on all the joint angles.

\section{APPENDIX \\ CONSIDERATIONS ABout SpRING SELECTION}

The stiffness and the initial length of the spring have to be set before running the optimisation algorithm. These parameters can be employed to define the size of pulleys or, when the analytical solution is unfeasible, to find a realistic shape. Knowing the maximum desired torque, $\tau_{\max }$, the size of the pulley is strictly related to the spring constant and initial length. Let assume to wrap the cable on a circular pulley. Under this assumption the maximum elongation of the spring is

$$
u_{\max }=\left(\theta_{f}-\theta_{i}\right) r+u_{0},
$$


TABLE V

SPRING PROPERTIES

\begin{tabular}{c|c|c|c}
\hline \hline Name & Symbol & Value & Unit \\
\hline \hline Wire diameter & $d$ & 13 & $\mathrm{~mm}$ \\
Mean coil diameter & $D$ & 87 & $\mathrm{~mm}$ \\
Active coils & $N_{a}$ & 15 & \\
Total coils & $N_{t}=N_{a}+2$ & 17 & \\
Required deflection & $L_{u}$ & 107 & $\mathrm{~mm}$ \\
Solid length & $L_{s}=d N_{t}$ & 221 & $\mathrm{~mm}$ \\
Pitch & $\left(L_{0}-2 d\right) / N_{a}$ & 20.13 & $\mathrm{~mm}$ \\
Young module & $E$ & 210000 & $\mathrm{MPa}$ \\
Shear module & $G$ & 81000 & $\mathrm{MPa}$ \\
Yield stress & $\sigma_{y}$ & 1300 & $\mathrm{MPa}$ \\
Poisson coefficient & $\nu$ & 0.3 & \\
\hline \hline
\end{tabular}

where $r$ is the pulley radius and $u_{0}$ the initial elongation. Thus the maximum torque acting on the pulley is computed as

$$
\tau_{\max }=k u_{\max } r=k r\left[\left(\theta_{f}-\theta_{i}\right) r+u_{0}\right] .
$$

Given the desired radius and a preliminary initial elongation, the order of magnitude of the spring constant is estimated from (41) as follows:

$$
k=\frac{\tau_{\max }}{\left(\theta_{f}-\theta_{i}\right) r^{2}+u_{0} r} .
$$

To verify this relation let assume that a radius of about $45 \mathrm{~mm}$ and a preliminary initial elongation of $0.015 \mathrm{~mm}$ are required. Then from (42) it is possible to estimate the spring stiffness as $k \approx 30000 \mathrm{~N} / \mathrm{m}$.

The properties of the candidate helical compression spring made of $52 \mathrm{CrMoV} 4$ are listed in Table V. As shown in [25], the elastic constant of the spring can be derived from

$$
k_{s}=\frac{d^{4} G}{8 D^{3} N_{a}}=29277 \frac{\mathrm{N}}{\mathrm{m}},
$$

where $D$ represents the mean coil diameter, $d$ the wire diameter, $G$ the shear module and $N_{a}$ the number of active coils. To obtain a motion in the range $\left[\theta_{i}, \theta_{f}\right]$ the required deflection of the spring is $L_{u}=107 \mathrm{~mm}$. Thus the free length of the spring is $L_{0}=$ $L_{u}+L_{s}=328 \mathrm{~mm}$ where $L_{s}$ is the solid length of the spring.

The maximum axial load is $F=k_{s} L_{u}=3133 \mathrm{~N}$. As shown in [25], the maximum stress of the designed helical spring is

$$
T_{\max }=\frac{8 F D}{\pi d^{3}}\left(\frac{4 C-1}{4 C-4}+\frac{0.615}{C}\right)=387 \mathrm{MPa},
$$

where $C=D / d=6.7$. The Von Mises stress of the critical point is equal to $\sigma^{\prime}=670 \mathrm{MPa}$. Considering a safety factor $s=1.5$, the condition $s \sigma^{\prime}<\sigma_{y}$ is verified and the spring design can be considered feasible.

\section{REFERENCES}

[1] V. Arakelian, "Gravity compensation in robotics," Adv. Robot., vol. 30, no. 2, pp. 79-96, 2016.

[2] Y. Tojo, P. Debenest, E. F. Fukushima, and S. Hirose, "Robotic system for humanitarian demining," in Proc. IEEE Int. Conf. Robot. Automat., 2004, pp. 2025-2030.
[3] J. Boisclair, P.-L. Richard, T. Laliberté, and C. Gosselin, "Gravity compensation of robotic manipulators using cylindrical halbach arrays," IEEE/ASME Trans. Mechatronics, vol. 22, no. 1, pp. 457-464, Feb. 2017.

[4] A. Rosyid, B. El-Khasawneh, and A. Alazzam, "Gravity compensation of parallel kinematics mechanism using torsional springs based on potential energy optimization," in Proc. 11th Int. Sympo. Mechatronics Appl., 2018, pp. $1-5$.

[5] G. Radaelli, R. Buskermolen, R. Barents, and J. Herder, "Static balancing of an inverted pendulum with prestressed torsion bars," Mechanism Mach. Theory, vol. 108, pp. 14-26, 2017.

[6] J. L. Herder, "Design of spring force compensation systems," Mechanism Mach. Theory, vol. 33, no. 1-2, pp. 151-161, 1998.

[7] J. Herder, "Energy-Free systems: Theory, conception and design of statically balanced spring mechanisms," 2001. [Online]. Available: https: //books.google.it/books?id=fBClAAAACAAJ

[8] V. Arakelian and Y. Zhang, "An improved design of gravity compensators based on the inverted slider-crank mechanism," J. Mechanisms Robot., vol. 11, no. 3, 2019, Art. no. 034501.

[9] H. Kim and J. Song, "Multi-dof counterbalance mechanism for a service robot ARM," IEEE/ASME Trans. Mechatronics, vol. 19, no. 6, pp. 1756-1763, Dec. 2014.

[10] S. Yun, J. Seo, J. Yoon, H. Song, Y. Kim, and Y. Kim, "3-dof gravity compensation mechanism for robot waists with the variations of center of mass," in Proc. IEEE/RSJ Int. Conf. Intelli. Robot. Syst., 2019, pp. 35653570.

[11] J. Reinecke, B. Deutschmann, A. Dietrich, and M. Hutter, "An anthropomorphic robust robotic torso for ventral/dorsal and lateral motion with weight compensation," IEEE Robot. Automat. Lett., vol. 5, no. 3, pp. 3876-3883, Jul. 2020.

[12] T. Rahman, R. Ramanathan, R. Seliktar, and W. Harwin, "A simple technique to passively gravity-balance articulated mechanisms," ASME J. Mech. Des., vol. 117, no. 4, pp. 655-658, Dec. 1995.

[13] T. Morita, F. Kuribara, Y. Shiozawa, and S. Sugano, "A novel mechanism design for gravity compensation in three dimensional space," in Proc. IEEE/ASME Int. Conf. Adv. Intell. Mechatronics, 2003, pp. 163-168.

[14] V. L. Nguyen, C.-Y. Lin, and C.-H. Kuo, "Gravity compensation design of planar articulated robotic arms using the gear-spring modules," $J$. Mechanisms Robot., vol. 12, no. 3, 2020, Art. no. 031014

[15] V. L. Nguyen, C.-Y. Lin, and C.-H. Kuo, "Gravity compensation design of delta parallel robots using gear-spring modules," Mechanism Mach. Theory, vol. 154, 2020, Art. no. 104046

[16] N. Ulrich and V. Kumar, "Passive mechanical gravity compensation for robot manipulators," in Proc. IEEE Int. Conf. Robot. Auto., 1991, pp. 15361541.

[17] I. Simionescu and L. Ciupitu, "The static balancing of the industrial robot arms: Part ii: Continuous balancing," Mechanism Mach. Theory, vol. 35 , no. 9, pp. 1299-1311, 2000.

[18] G. Endo, H. Yamada, A. Yajima, M. Ogata, and S. Hirose, "A passive weight compensation mechanism with a non-circular pulley and a spring," in Proc. IEEE Int. Conf. Robot. Auto., 2010, pp. 3843-3848.

[19] D. Fedorov and L. Birglen, "Differential noncircular pulleys for cable robots and static balancing," J. Mechanisms Robot., vol. 10, no. 6, 2018, Art. no. 061001 .

[20] B. Kim and A. D. Deshpande, "Design of nonlinear rotational stiffness using a noncircular pulley-spring mechanism," J. Mechanisms Robot., vol. 6, no. 4, 2014, Art. no. 041009 .

[21] J. W. Rutter, Geometry of Curves, London, U.K.: Chapman \& Hall/CRC, 2000.

[22] A. J. McPhate, "Function generation with band mechanisms," J. Mechanisms, vol. 1, no. 1, pp. 85-94, 1966.

[23] M. Grant and S. Boyd, "CVX: Matlab Software for Disciplined Convex Programming, version 2.1," Mar. 2014. [Online]. Available: http://cvxr. com/cvx.

[24] M. Grant and S. Boyd, "Graph implementations for nonsmooth convex programs," in Recent Advances in Learning and Control, ser. Lecture Notes in Control and Information Sciences, V. Blondel, S. Boyd, and H. Kimura, Eds. Springer-Verlag Limited, 2008, pp. 95-110.

[25] J. E. Shigley, Shigley's Mechanical Engineering Design. Tata McGrawHill Education, 2011. 